\title{
The Cores for Fuzzy Games Represented by the Concave Integral
}

\author{
Jinhui Pang ${ }^{1}$ and Shujin $\mathrm{Li}^{2}$ \\ ${ }^{1}$ Library, Beijing Institute of Technology, Beijing 100081, China \\ ${ }^{2}$ Department of Information Management, The Central Institute for Correctional Police, Baoding 071000, China
}

Correspondence should be addressed to Jinhui Pang; pangjinhui69@126.com

Received 31 October 2013; Accepted 10 January 2014; Published 13 March 2014

Academic Editor: Shusen Ding

Copyright (C) 2014 J. Pang and S. Li. This is an open access article distributed under the Creative Commons Attribution License, which permits unrestricted use, distribution, and reproduction in any medium, provided the original work is properly cited.

\begin{abstract}
We propose a new fuzzy game model by the concave integral by assigning subjective expected values to random variables in the interval $[0,1]$. The explicit formulas of characteristic functions which are determined by coalition variables are discussed in detail. After illustrating some properties of the new game, its fuzzy core is defined; this is a generalization of crisp core. Moreover, we give a further discussion on the core for the new games. Some notions and results from classical games are extended to the model. The nonempty fuzzy core is given in terms of the fuzzy convexity. Our results develop some known fuzzy cooperative games.
\end{abstract}

\section{Introduction}

In crisp cooperative game, gains from a coalition are supposed to be of certainty so that the solution concepts are also definitive which determine allocations of the total benefit from cooperation to the players. However, the cooperation is full of uncertainty.

The fuzzy game theory also deals with the problems of how to describe fuzzy coalitions, how to represent available fuzzy payoffs, and how to divide it among the various players. In Aubin [1] and Butnariu [2] fuzzy game, the characteristic function was an aggregated worth of the coalitions profits, which depended on the degree of participations of players in a coalition. Tsurumi et al. [3] proposed a class of fuzzy games using the concept of Choquet integrals.

The cooperative games, which lack precision game data, had been investigated by stochastic theory (see Granot [4] and Fernandez et al. [5]) In some situations, there is no reliable information on probability distributions and other aspects of the problems. Consequently, it is reasonable to adopt the fuzzy theory to constructing various fuzzy game models (see Zadeh [6], Mareš [7], Dubois and Prade [8]).

Mareš $[9,10]$ and Vlach [11] suggested that the uncertainty value of the characteristic function was also associated with a game. By this assumption, the values assigned to coalitions were also fuzzy quantities even though the domain of the characteristic function of fuzzy games remained to be the same as crisp games.

Borkotokey [12] considered a cooperative game with fuzzy coalitions and fuzzy characteristic function simultaneously, whose characteristic functions were a fuzzy value which mapped the set of real numbers to the closed interval $[0,1]$.

Nowadays, the fuzzy games are mainly introduced in two ways. One is games with fuzzy payoffs and the coalitions are still crisp game coalitions. Another is games with fuzzy coalitions in which players partly take part in a coalition so as to form a fuzzy coalition but exact benefits from the fuzzy coalition can be attained.

After fuzzy games were defined, their solution concepts have been studied by many scholars. The firstly defined fuzzy Shapley function by Butnariu [13] showed a specific formula on a limited class of fuzzy games with proportional values. But it was neither monotone nondecreasing nor continuous with regard to rates of players' participation. Later, Butnariu and Kroupa [14] gave an analogous Shapley function on fuzzy games with weight function. Following Butnariu's way, Tsurumi et al. defined Shapley function on the fuzzy game with Choquet integral form, which was both monotone nondecreasing and continuous with regard to rates of players' participation rates because of the advantage properties of Choquet integral. In fact, the core for fuzzy games is another important solution concept which was focused on 
by Tijs et al. [15]. With the development of fuzzy cooperative game theory, many extended solutions of some fuzzy games, which are homogeneous or one-to-one of crisp game, draw much more attention of researchers.

In many game activities, players estimate game utility according to what is referred to as an uncertainty aversion. In this way, integrals with fuzzy measures should be suitable to model fuzzy games. Dow and Werlang $[16,17]$ applied the Choquet integral to game theory and finance. The integral theory had made use of the concavification of a cooperative game that appeared in Weber [18] and later in Azrieli and Lehrer [19]. Lehrer [20] proposed a new integral for capacities which differs from the Choquet integral on nonconvex capacities and discussed its properties in detail.

In fuzzy game situations, fuzzy capacities assign subjective expected values to some coalitions but not to all. Inspired by Lehrer's new integral, we introduce a new cooperative game form by the new integral with respect to fuzzy capacity. Further, it has been shown that the game defined by Tsurumi et al. is a special case when the fuzzy capacity satisfies convex in our new class of games. We have also defined a fuzzy core in order to provide a solution concept for the new proposed game.

The paper will be organized as follows. In Section 2, we introduce the concepts of crisp cooperative game and its imputation. In Section 3, some basic concepts of games with fuzzy coalitions and several game models, such as Butnariu and Tsurumi fuzzy games, will be given. In Section 4 , we define a new fuzzy game by the concave integral and its several equal integral representations which described the fuzzy characteristic functions of the cooperative game will also be given. Meanwhile, some properties are discussed. We propose fuzzy core concept for the new game in Section 5 and its nonempty condition is given based on the fuzzy convexity. Moreover, we will give a calculating way of a fuzzy core. Finally, some conclusions appeared in Section 6.

\section{Crisp Cooperative Game and Its Imputation}

We consider cooperative games with a finite set of players $N=\{1,2, \ldots, n\}$ who may consider different cooperation possibilities. The set $N$ is the grand set and any subset $S$ of $N$ can be seen as the grand coalition relative to $S \subseteq T$. The notation $P(S)$ is the family of all crisp subsets (subcoalitions) of $S \subseteq N$.

A crisp cooperative game on player set $N$ is denoted by $v$, where the characteristic function $v: P(N) \rightarrow$ $R_{+} \cup\{0\}$ with $v(\phi)=0$ and $v(S)(S \in P(N))$ is the worth of coalition $S$ which can be seen as the global utility when the players work in the coalition $S$ together. The class of crisp games with player set $N$ is denoted by $G(N, v)$.

The game $v \in G(N, v)$ is said to be convex when

$$
v(S \cup T)+v(S \cap T) \geq v(S)+v(T), \quad \forall S, T \in P(N)
$$

If $S$ and $T$ are disjoint crisp coalitions in convex game $v \in$ $G(N, v)$, that is,

$$
v(S \cup T) \geq v(S)+v(T), \quad \forall S, T \in P(N), \quad S \cap T=\phi,
$$

then the game is said to be superadditive and we denote all the superadditive crisp cooperative games by $G_{0}(N, v)$.

Definition 1. An imputation for a crisp cooperative game $v \in$ $G(N, v)$ is a vector $x=\left(x_{1}, x_{2}, \ldots, x_{n}\right) \in R_{+}^{n} \cup\{0\}$ satisfying

(1) $\sum_{i \in N} x_{i}=v(N)$.

(2) $x_{i} \geq v(i), \forall i \in N$.

A set of imputation of $v \in G(N, v)$ is nonempty, and then the game is a superadditive crisp cooperative game, for example, $v \in G_{0}(N, v)$.

The important solutions, such as the core and Shapley value, are imputation for a crisp cooperative game. The core on crisp game $v \in G_{0}(N, v)$ is a convex set including all undominated imputations such that

$$
\begin{aligned}
C(v, N)=\left\{x \in R_{+}^{n} \mid \sum_{i \in N} x_{i}=v(N),\right. \\
\\
\left.\sum_{i \in S} x_{i} \geq v(S), \forall S \in P(N)\right\} .
\end{aligned}
$$

The Shapley value of player $i$ is a probabilistic value and has a unique expression, which can be considered as the expectation of his marginal contribution $v(T \cup i)-v(T)$ to any coalition $T \in P(N-i)$, which is

$$
\operatorname{Sh}_{i}(v)=\sum_{T \subseteq N \backslash i} \frac{(|N|-|T|-1)|T| !}{|N| !}[v(T \cup i)-v(T)],
$$

where $|\cdot|$ is the cardinality of a coalition.

Note that when $v \in G_{0}(N, v)$, then the Shapley vector

$$
\operatorname{Sh}(v)=\left(\operatorname{Sh}_{1}(v), \operatorname{Sh}_{2}(v), \ldots, \operatorname{Sh}_{n}(v)\right) \in C(v, N) \text {. }
$$

\section{Some Concepts of Game with Fuzzy Coalitions}

A fuzzy coalition $\widetilde{S}$ is a fuzzy subset of the finite set $N=$ $\{1,2, \ldots, n\}$, which assigned a real valued function from $N$ to $[0,1]$. In other words, a fuzzy coalition can be represented by a vector $s=\left(s_{1}, s_{2}, \ldots, s_{n}\right)$, where $s_{i} \in[0,1]$ is a constant denoting the membership grade of player $i$ in the fuzzy coalition $\widetilde{S}$. Of course, the set $N$ is the grand coalition and $\phi$ is the empty coalition. It corresponds to the situation where the players participate fully in $\widetilde{S}$; that is, each element of the level vector $s=\left(s_{1}, s_{2}, \ldots, s_{n}\right)$ has participation level 1 , and the players outside $\widetilde{S}$ are not involved at all; that is, they have participation level 0 .

The set of all fuzzy coalitions in $N$ is denoted by $L(N)$. The support set of fuzzy coalition $\widetilde{S} \in L(N)$ defined by 
$\operatorname{Supp}(\widetilde{S})=\left\{i \in N \mid s_{i}>0\right\}$ which is a subset of $N$; its level subset denoted by $\widetilde{S}$ is $[\widetilde{S}]_{t}=\left\{i \in N \mid s_{i} \geq t\right\}$. For $r \in[0,1]$, $r$-section for $\widetilde{S}$ is the set $\widetilde{S}^{r}=\left\{i \mid i \in N, s_{i}=r\right\}$ which means a player set with the same level. Suppose the fuzzy coalition $\widetilde{S}$ and $\widetilde{U}$ have vector $s$ and $u$ respectively, then $s_{i} \leq u_{i}(\forall i \in N)$ means that $\widetilde{S} \subseteq \widetilde{U}$. A fuzzy cooperative game $\widetilde{v}$ is the function $\widetilde{v}: L(N) \rightarrow R_{+} \cup\{0\}$ with $v(\phi)=0 . \widetilde{G}(N, \widetilde{v})$ denotes the class of all fuzzy games $\widetilde{v}$.

In this paper, we assume that every of the fuzzy coalitions maps into the lattice $([0,1], \wedge, \vee)$, where $\wedge$ and $\vee$ are the minimum and maximum operators, respectively.

For any fuzzy coalition $\widetilde{S}, \widetilde{U} \in L(N)$, the union of two fuzzy coalitions $\widetilde{S}$ and $\widetilde{U}$ is denoted as $\widetilde{S} \cup \widetilde{U}$ which satisfies

$$
(\widetilde{S} \cup \widetilde{U})(i)= \begin{cases}s_{i} \vee u_{i}, & i \in \operatorname{Supp} \widetilde{S} \cup \operatorname{Supp} \widetilde{U} \\ 0, & \text { others. }\end{cases}
$$

Similarly, the intersection $\widetilde{S} \cap \widetilde{U}$ satisfies

$$
(\widetilde{S} \cap \widetilde{U})(i)= \begin{cases}s_{i} \wedge u_{i}, & i \in \operatorname{Supp} \widetilde{S} \cap \operatorname{Supp} \widetilde{U}, \\ 0, & \text { others. }\end{cases}
$$

Corresponding to the convex game in crisp game, the convex fuzzy game is defined as follows.

Definition 2. A function $\widetilde{v}: L(N) \rightarrow R_{+} \cup\{0\}$ is said to be fuzzy convex, if

$$
v(\widetilde{S})+v(\widetilde{U}) \leq v(\widetilde{S} \cup \widetilde{U})+v(\widetilde{S} \cap \widetilde{U}),
$$

for all $\widetilde{S}, \widetilde{U} \in L(N)$.

Definition 3. $\widetilde{v} \in \widetilde{G}(N, \widetilde{v})$ is said to be superadditive, if

$$
v(\widetilde{S} \cup \widetilde{U}) \geq v(\widetilde{S})+v(\widetilde{U}),
$$

for all $\widetilde{S}, \widetilde{U} \in L(N)$ and $\widetilde{S} \cap \widetilde{U}=\phi$.

In fuzzy game literature, there are several game models which were aggregated function on fuzzy level coalitions, such as Butnariu game, Butnariu and Kroupa game, and Tsurumi game. Based on the definition of $r$-section, Butnariu [13] proposed a fuzzy game with proportional values which was weighted by the participation level $r$.

Definition 4. The game $v^{p} \in \widetilde{G}(N, \widetilde{v})$ is said to be with proportional values if and only if

$$
v^{p}(\widetilde{S})=\sum_{r \in[0,1]} v\left(\widetilde{S}^{r}\right) \cdot r, \quad \forall \widetilde{S} \in L(N) .
$$

It should be noted that there is a one-to-one correspondence between a crisp game and a fuzzy game with proportional values, because the characteristic function is a linear aggregation function which is a weighted average on the sets with the same participation levels. For the sake of simplicity, we will denote the fuzzy game with proportional values as the notation $G_{p}(N, v)$.

As the extension of games with proportional value, Butnariu and Kroupa [14] proposed a class of games with weight function.
Definition 5. The game $v^{\psi}: L(N) \rightarrow R$ satisfying $v(\phi)=0$ is called a fuzzy game with weight function if and only if

$$
v^{\psi}(\widetilde{S})=\sum_{r \in[0,1]} \psi(r) v\left(\widetilde{S}^{r}\right),
$$

where $\psi:[0,1] \rightarrow R$ is a function with the properties $\psi(r)=$ $0 \Leftrightarrow r=0$ and $\psi(1)=1$.

The set of games with weight functions is denoted by $G_{\psi}(N, v)$. If $\psi(r)=r$, then the fuzzy game $v \in G_{\psi}(N, v)$ is equivalent to the game $v \in G_{p}(N, v)$.

It is obvious that the characteristic function of a cooperative game with proportional values or weight functions is a linear aggregation function. For any fuzzy game $v \in$ $G_{\psi}(N, v)$, there is no excess of any two players with different participation levels, and the payoffs of a fuzzy coalition are only a simple accumulation of utility created by players with the same participation level. These two fuzzy game models defined by Butnariu cannot embody the interaction among players with different participation levels.

After considering Butnariu's approach, Tsurumi et al. thought that most of this class games were neither monotone nondecreasing nor continuous with regard to rates of players' participations although crisp games are often considered to be monotone nondecreasing. In other words, these games cannot be regarded as quite natural. Tsurumi defined a payoff function and a fuzzy population monotonic allocation scheme as extensions of imputation and population monotonic allocation scheme. The following definitions and theorems were introduced.

Definition 6. Given $\widetilde{S} \in L(N)$, let $Q(\widetilde{S})=\left\{s_{i} \mid s_{i}>0, i \in N\right\}$ and let $q(\widetilde{S})$ be the cardinality of $Q(\widetilde{S})$. The elements in $Q(\widetilde{S})$ are rewritten by the increasing order as $h_{1}<h_{2}<\cdots<h_{q(\tilde{S})}$. Then, a game $v^{C h}: L(N) \rightarrow R$ is said to be a fuzzy game with Choquet integral form if and only if the following holds:

$$
v^{C h}(\widetilde{S})=\sum_{l=1}^{q(\widetilde{S})} v\left([\widetilde{S}]_{h_{l}}\right) \cdot\left(h_{l}-h_{l-1}\right)
$$

for any $\widetilde{S} \in L(N)$, where $h_{0}=0$.

It is apparent that the fuzzy game model proposed by Tsurumi, which incorporated the notion of vague expectation along with fuzzy coalitions, is a Choquet integral of the function $h$ with respect to $v$ derived from level set. We note that there is also a one-to-one correspondence between a crisp game and a fuzzy game with Choquet integral form. For the sake of simplicity, a fuzzy game with Choquet integral form is denoted by $G_{\mathrm{Ch}}(N, v)$.

In the above definition, let a set $Q(\widetilde{S}) \subseteq\left\{k_{1}, k_{2}, \ldots, k_{m}\right\}$ such that $0=k_{0} \leq k_{1}<k_{2}<\cdots<k_{m} \leq 1$; then

$$
v^{C h}(\widetilde{S})=\sum_{l=1}^{m} v\left([\widetilde{S}]_{k_{l}}\right) \cdot\left(k_{l}-k_{l-1}\right) .
$$

Tsurumi et al. had proved that the game $v \in G_{\mathrm{Ch}}(N, v)$ has the following properties. 
Proposition 7. Let $v \in G_{C h}(N, v)$, for any $\widetilde{K}, \widetilde{U} \in L(N)$ and $\widetilde{K} \subseteq \widetilde{U}$; then the following holds:

$$
v(\widetilde{K}) \leq v(\widetilde{U}) .
$$

Proposition 8. Let $v \in G_{C h}(N, v)$; define the distance $H(\widetilde{K}, \widetilde{U})=\max _{i \in N}\left|k_{i}-u_{i}\right|$ for any $\widetilde{K}, \widetilde{U} \in L(N)$; then $v$ is continuous.

Proposition 9. Let $v \in G_{C h}(N, v)$ and $\widetilde{S}, \widetilde{T} \in L(N)$ such that $v(\widetilde{S})=v(\widetilde{T})$ if and only if

$$
v\left([\widetilde{S}]_{h}\right)=v\left([\widetilde{T}]_{h}\right) \quad \text { for any } h \in(0,1] .
$$

\section{The Concave Integral Representation for Fuzzy Cooperative Game}

The characteristic function of fuzzy game with Choquet integral form defined according to the Choquet integral is an expected value of utility with respect to a nonadditive probability distribution. Players may choose the act that maximizes the expected utility so that the one that achieves the maximum of the respective value is chosen. Since a particular decomposition of $Q(\widetilde{S})$ rather than all possible decompositions is used for the calculation of the Choquet integral, the value $v^{\mathrm{Ch}}(\widetilde{S})$ may not be a more reasonable outcome, while the method related to the concave integral seems to be more suitable to measuring the productivity of a coalition.

Let $N$ be a finite set $(|N|=n)$; a capacity $\mu$ over $N$ is a function $\mu: P(N) \rightarrow R^{+} \cup\{0\}$ such that $S \subseteq T \subseteq N$ implies $\mu(S) \leq \mu(T)$ with $\mu(\phi)=0$. A random variable over $N$ is a function $X: N \rightarrow R$ and a random variable is nonnegative if $X(i) \geq 0$ for every $i \in N$.

Definition 10. Let $X$ be a random variable; a subdecomposition of $X$ is a finite summation $\sum_{i=1}^{k} \alpha_{i} 1_{A_{i}}$ that satisfies

$$
\sum_{i=1}^{k} \alpha_{i} 1_{A_{i}} \leq X \quad\left(\alpha_{i} \geq 0\right), A_{i} \subseteq N(i=1,2, \ldots, k) .
$$

Definition 11. Let $\mu$ be a capacity over $N$ and let $X$ be a nonnegative random variable; define the concave integral as

$$
\int^{\mathrm{Cav}} X d \mu=\min \{f(X)\}
$$

where the minimum is taken all over concave and homogeneous functions $f: R_{+}^{n} \rightarrow R$ such that $f\left(1_{A}\right) \geq v(A)$, for every $A \subseteq N$, where $1_{A}$ is the indicator of $A$ which is the random variable that takes the value 1 over $A$ and the value 0 otherwise.

Let $\mu$ and $\omega$ be two capacities, if $\mu \geq \omega$ implies that $\mu(S) \geq$ $\omega(S)$ for every $S \subseteq N$. Lehrer had proved the concave integral properties.
Proposition 12. For every nonnegative $X$ defined over $N$,

$$
\begin{gathered}
\int^{C a v} X d \mu=\max \left\{\sum_{A \subseteq N} \alpha_{A} \mu(A), \sum_{A \subseteq N} \alpha_{A} 1_{A}=X, \alpha_{A} \geq 0\right\}, \\
\int^{C a v} X d \mu=\min \int X d P,
\end{gathered}
$$

where $P$ is additive and $P \geq \mu$.

Note that the capacities $P$ need not be a probability distribution and $P(N)=\mu(N)$ is not necessary.

The Choquet integral of nonnegative $X$ with respect to a capacity $\mu$ is defined by

$$
\int^{\mathrm{Ch}} X d \mu=\sum_{i=1}^{n}\left(X_{\sigma(i)}-X_{\sigma(i-1)}\right) \mu\left(Q_{i}\right),
$$

where $\sigma$ is a permutation on $N$ such that $0=X_{\sigma(0)} \leq X_{\sigma(1)} \leq$ $X_{\sigma(2)} \leq \cdots \leq X_{\sigma(n)}$ and $Q_{i}=\{\sigma(i), \sigma(i+1), \ldots, \sigma(n)\}$.

Let $\alpha_{i}=X_{\sigma(i)}-X_{\sigma(i-1)}$; note that $X=\sum \alpha_{i} 1_{Q_{i}}$ is a decomposition of $X$. That is to say, the Choquet integral is defined under a special decomposition of $X$. By contrast, all possible decompositions are allowed in the concave integral. It means that $\int^{\mathrm{Ch}} X d \mu \leq \int^{\mathrm{Cav}} X d \mu$ for any $X$. In addition, $\int^{\mathrm{Ch}} X d \mu=\int^{\mathrm{Cav}} X d \mu$ if and only if $\mu$ is convex.

In fuzzy game literature, many researchers devote lots work to searching for a better expression of fuzzy game. But most of them were usually limited to the participation levels of players and the payoffs of crisp coalitions.

It should be noted that there are some fuzzy coalitions whose payoffs cannot be expressed by crisp coalition values and participation levels. As a result, their method of constructing fuzzy characteristic function, which is only limited to some special game, will be invalid in many game situations. Inspired by better properties of the concave integral, we follow the method of Tsurumi game to define a new class fuzzy game, where Tsurumi game can take a special case as the proposed new game.

Letting $N$ be a finite players set, the vector $x=$ $\left(x_{1}, x_{2}, \ldots, x_{n}\right), x_{i} \in[0,1]$ is a random variable over $N$ which represents a fuzzy coalition, namely, coalition variable, and $x_{i}$ is a level variable fraction of player $i$ in the fuzzy coalition. All the coalition variables set of $x$ over $N$ is denoted by $L(x)=\left\{x \mid x=\left(x_{1}, x_{2}, \ldots, x_{n}\right), x_{i} \in[0,1]\right\}$. Similarly, for any $A \subseteq N$, all subcoalitions set is denoted by $L(A)=\{x \mid$ $\left.x=\left(x_{1}, x_{2}, \ldots, x_{|A|}\right), i \in A\right\}$.

We will define a new class of fuzzy games which has its good properties, as will be shown in what follows.

Definition 13. Let $v \in G(N, v)$. Given $\widetilde{S} \in L(N)$, let $s=$ $\left(s_{1}, s_{2}, \ldots, s_{n}\right) \in L(x)$ be a coalition variable over $N$; then a game $v^{\mathrm{Cav}}: L(N) \rightarrow R_{+} \cup\{0\}$ is said to be a fuzzy game with concave integral form, if and only if

$$
v^{\mathrm{Cav}}(\widetilde{S})=\int^{\mathrm{Cav}} s d v=\min \{f(s)\},
$$

where $f: R_{+}^{n} \rightarrow R$ and $f\left(1_{T}\right) \geq v(T)$ for every $T \subseteq N$. 
Remark 14. In Definition 13, $\min \{f(s)\}$ is the characteristic function of the fuzzy games with concave integral form and the minimum is taken over all concave and homogeneous functions $f: R_{+}^{n} \rightarrow R$. Since the domain of the minimum of the family function of concave and homogeneous functions $f$ is $R_{+}^{n}$, so $v^{\mathrm{Cav}}(\widetilde{S})$ is concave and homogeneous and the class of the fuzzy games with concave integral form is nonempty.

We denote all the fuzzy games with concave integral form as $G_{\text {Cav }}(N, v)$. In fact, from Definition 10, the characteristic function of the fuzzy game with concave integral form can be gained by the subdecomposition of $s$ and the characteristic function of subdecomposition crisp coalitions.

Let $v \in G(N, v)$. Given $\widetilde{S} \in L(N)$, let $s=\left(s_{1}, s_{2}, \ldots, s_{n}\right) \in$ $L(x)$ be a coalition variable over $N$; then a game $v^{\mathrm{Cav}}$ : $L(N) \rightarrow R_{+} \cup\{0\}$ can also be calculated by

$$
\begin{aligned}
v^{\mathrm{Cav}}(\widetilde{S}) & =\int^{\mathrm{Cav}} s d v=\max \sum_{A_{i} \subseteq N} \alpha_{A_{i}} v\left(A_{i}\right) \\
\text { s.t. } & \left\{\begin{array}{l}
\sum_{i=1}^{n} \alpha_{A_{i}} 1_{A_{i}}=s \\
A_{i} \subseteq N \quad(i=1,2, \ldots, n), \\
\alpha_{A_{i}} \in\left\{s_{1}, s_{2}, \ldots, s_{n}\right\} .
\end{array}\right.
\end{aligned}
$$

Let $\left.Q(\widetilde{S})=\left\{s_{i} \mid s_{i}>0, i \in N\right\}\right\}$ and the cardinality $|\widetilde{S}|=$ $q(\widetilde{S})$; the above inequality is equal to

$$
\begin{aligned}
v^{\mathrm{Cav}}(\widetilde{S})= & \int^{\mathrm{Cav}} s d v=\max \sum_{A_{i} \subseteq N} \alpha_{A_{i}} v\left(A_{i}\right) \\
\text { s.t. } & \left\{\begin{array}{l}
\sum_{i=1}^{|\widetilde{S}|} \alpha_{A_{i}} 1_{A_{i}}=s \\
\alpha_{A_{i}} \in\left\{s_{1}, s_{2}, \ldots, s_{n}\right\} \\
i=1,2, \ldots,|\widetilde{S}|
\end{array}\right.
\end{aligned}
$$

Lemma 15. Let $v \in G(N, v)$. Given $\widetilde{S} \in L(N)$, let $s=$ $\left(s_{1}, s_{2}, \ldots, s_{n}\right) \in L(x)$ be a coalition variable over $N$; then a game $v^{\text {Cav }}: L(N) \rightarrow R_{+} \cup\{0\}$ is

$$
\begin{gathered}
v^{C a v}(\widetilde{S})=\int^{C a v} s d v=\max \sum_{A_{i} \subseteq N} s_{i} v\left(A_{i}\right) \\
\text { s.t. }\left\{\sum_{i=1}^{n} \alpha_{A_{i}} 1_{A_{i}}=s .\right.
\end{gathered}
$$

Example 16. Suppose that three workers work on a joint project; let players set $N=\{1,2,3\}$ and let $v$ be a characteristic function on $N$ which is joint workers' output. $v(1)=v(2)=v(3)=2, v(1,3)=8, v(1,2)=9, v(2,3)=5$, and $v(1,2,3)=10$. Let the fuzzy coalition $s=(1,0.4,0.6)$; then, by (21), we have that

$$
\begin{gathered}
\qquad v^{\mathrm{Cav}}(\widetilde{S})=\int^{\mathrm{Cav}} s d v=\max \sum_{A_{i} \subseteq N} \alpha_{A_{i}} v\left(A_{i}\right) \\
\text { s.t. }\left\{\begin{array}{l}
\sum_{i=1}^{k} \alpha_{A_{i}} 1_{A_{i}}=(1,0.4,0.6), \\
A_{i} \in\{\{1\},\{2\},\{3\},\{1,3\},\{1,2\},\{2,3\},\{1,2,3\}\}, \\
\alpha_{A_{i}} \in\{0.4,0.6,1\} .
\end{array}\right.
\end{gathered}
$$

Hence,

$$
\begin{array}{cc}
A_{1}=\{1,2\}, & A_{2}=\{1,3\}, \\
\alpha_{A_{1}}=0.4, & \alpha_{A_{2}}=0.6 .
\end{array}
$$

So

$$
v^{\text {Cav }}(\widetilde{S})=0.4 \times 9+0.6 \times 8=8.4 .
$$

However, by the method given by Tsurumi et al., we rearrange the factor of fuzzy coalition variable $s=(1,0.4,0.6)$ such that $0.4<0.6<1$. We get the level sets $[\widetilde{S}]_{0.4}=\{1,2,3\},[\widetilde{S}]_{0.6}=$ $\{1,3\}$, and $[\widetilde{S}]_{1}=\{1\}$; then we have

$$
\begin{aligned}
v^{C}(\widetilde{S})= & v\left([\widetilde{S}]_{0.4}\right) \times 0.4+v\left([\widetilde{S}]_{0.6}\right) \times(0.6-0.4) \\
& +v\left([\widetilde{S}]_{1}\right) \times(1-0.6) \\
= & v(1,2,3) \times 0.4+v(1,3) \times 0.2+v(1) \times 0.4=6.4 .
\end{aligned}
$$

It is easy to see that the outputs of the fuzzy game are different and $v^{C h}(\widetilde{S})<v^{\mathrm{Cav}}(\widetilde{S})$. Therefore, the characteristic function given by Tsurumi et al. is not the maximal product such that Tsurumi fuzzy game is not more suitable than the proposed method by the concave integral in some situations.

We know that $v^{\mathrm{Cav}}(\widetilde{S})=\int^{\mathrm{Cav}} s d v$ is the maximum of the values $\sum_{i=1}^{k} \alpha_{A_{i}} \mu\left(A_{i}\right)$ among all possible decompositions of $\widetilde{S}$ with the coalition variable $s$. The fuzzy game given by the concave integral imposes no restriction over the decompositions being used; that is, all possible decompositions are taken into account when considering the maximum. Although the fuzzy game given by the Choquet integral can also be expressed in terms of decompositions, unlike the concave integral, Choquet integral instead does impose restrictions.

We say that two subsets $A$ and $B$ of $N$ are nested if either $A \subseteq B$ or $B \subseteq A$. We recall the traditional definition of the Choquet integral. Let $\sigma$ be a permutation on $N$, such that $X_{\sigma(1)} \leq X_{\sigma(2)} \leq \cdots \leq X_{\sigma(n)}$. The Choquet integral is the summation $\sum_{i=1}^{n}\left(X_{\sigma(i)}-X_{\sigma(i-1)}\right) \mu\left(Q_{i}\right)$ in which $Q_{i}=\{\sigma(i), \ldots, \sigma(n)\}$. Therefore, the fuzzy game with Choquet integral form is the maximum of $\sum_{i=1}^{k} \alpha_{A_{i}} v\left(1_{A_{i}}\right)$ among all decompositions in which every $A_{i}$ and $A_{j}$ are nested for any $i, j$. 
Lemma 17. Let $v \in G(N, v)$. Given $\widetilde{S} \in L(N)$, let $s=$ $\left(s_{1}, s_{2}, \ldots, s_{n}\right), s_{i} \in[0,1]$.

One has

$$
v^{C h}(\widetilde{S})=\int^{C h} s d v=\max \sum_{A_{i} \subseteq N} \alpha_{A_{i}} v\left(A_{i}\right),
$$

where $A_{i} \supseteq A_{j}(\forall i<j)$.

The fuzzy game defined by the Choquet integral is the maximum of $\sum_{i=1}^{k} \alpha_{A_{i}} v\left(A_{i}\right)$ over all decompositions in which every $A_{i}$ and $A_{j}$ are nested. It is evident that $v^{\mathrm{Ch}}(\widetilde{S}) \leq v^{\mathrm{Cav}}(\widetilde{S})$.

The game $v \in G_{\text {Cav }}(N, v)$ has the following properties.

Proposition 18. Let $v \in G_{C a v}(N, v)$, for any $\widetilde{K}, \widetilde{U} \in L(N)$ and $\widetilde{K} \subseteq \widetilde{U}$; then the following holds

$$
v(\widetilde{K}) \leq v(\widetilde{U}) .
$$

Proof. Let $\widetilde{K}, \widetilde{U} \in L(N)$ with the fuzzy coalition variables $k=$ $\left(k_{1}, k_{2}, \ldots, k_{n}\right)$ and $u=\left(u_{1}, u_{2}, \ldots, u_{n}\right)$.

Since $\widetilde{K} \subseteq \widetilde{U}$, we get $k_{i} \leq u_{i}(i=1,2, \ldots, n)$ and $\int^{\mathrm{Cav}} k d v \leq \int^{\mathrm{Cav}} u d v$.

Hence, $v(\widetilde{K}) \leq v(\widetilde{U})$.

Proposition 19. Let $v \in G_{C a v}(N, v)$; define the distance $H(\widetilde{K}, \widetilde{U})=\max _{i \in N}\left|k_{i}-u_{i}\right|$ for any $\widetilde{K}, \widetilde{U} \in L(N)$; then $v$ is continuous.

Proof. Let $\widetilde{K}, \widetilde{U} \in L(N)$ with the fuzzy coalition variables $k=$ $\left(k_{1}, k_{2}, \ldots, k_{n}\right)$ and $u=\left(u_{1}, u_{2}, \ldots, u_{n}\right)$.

Suppose $\widetilde{K} \rightarrow \widetilde{U}$ such that $h=H(\widetilde{K}, \widetilde{U})=\max _{i \in N} \mid k_{i}-$ $u_{i} \mid \rightarrow 0$; denote $\delta=o(|h|)=\left(\delta_{1}, \delta_{2}, \ldots, \delta_{n}\right)$; then $k_{i}=u_{i}+$ $\delta_{i}(\forall i \in N)$.

So,

$$
\begin{aligned}
& v^{\text {Cav }}(\widetilde{K})-v^{\text {Cav }}(\widetilde{U})=\int^{\text {Cav }}(k-u) d v \\
&=\int^{\text {Cav }} o(|h|) d v \\
&=\int^{\text {Cav }} \delta d v \\
&=\max \sum_{A_{i} \subseteq N} \alpha_{A_{i}} v\left(A_{i}\right) \\
& \text { s.t. }\left\{\sum_{A_{i} \subseteq N} \alpha_{A_{i}} 1_{A_{i}}=\delta,\right. \\
& \alpha_{A_{i}} \in\left\{\delta_{1}, \delta_{2}, \ldots, \delta_{n}\right\} .
\end{aligned}
$$

That is, $\int^{\text {Cav }}(k-u) d v=0$ when $o(|h|) \rightarrow 0$.

Hence, if $H(\widetilde{K}, \widetilde{U})=\max _{i \in N}\left|k_{i}-u_{i}\right| \rightarrow 0$, then $v(\widetilde{K}) \rightarrow$ $v(\widetilde{U})$. That is, $v$ is continuous.

Proposition 20. Let $v \in G_{C a v}(N, v)$ and $\widetilde{S}, \widetilde{T} \in L(N)$ such that $v(\widetilde{S})=v(\widetilde{T})$ if and only if

$$
v\left([\widetilde{S}]_{h}\right)=v\left([\widetilde{T}]_{h}\right) \quad \text { for any } h \in[0,1] .
$$

Proof. Let $v \in G_{\mathrm{Cav}}(N, v)$ and $\widetilde{S}, \widetilde{T} \in L(N), v(\widetilde{S})=v(\widetilde{T})$; we get $\int^{\mathrm{Cav}} s d v=\int^{\mathrm{Cav}} u d v$; that is,

$$
v^{\text {Cav }}(\widetilde{S})=\max \sum_{A_{i} \subseteq N} \alpha_{A_{i}} v\left(A_{i}\right)
$$

$$
\text { s.t. }\left\{\begin{array}{l}
\sum_{i=1}^{n} \alpha_{A_{i}} 1_{A_{i}}=u, \\
A_{i} \subseteq N \quad(i=1,2, \ldots, n), \\
\alpha_{A_{i}} \in\left\{u_{1}, u_{2}, \ldots, u_{n}\right\} .
\end{array}\right.
$$

For any $i$, denote $\alpha_{A_{i}}=h_{i}, h_{i} \in[0,1]$; then

$$
\begin{array}{r}
\max \sum_{A_{i} \subseteq N} h_{i} v\left([\widetilde{S}]_{h_{i}}\right)=\max \sum_{A_{i} \subseteq N} h_{i} v\left([\widetilde{U}]_{h_{i}}\right), \\
h_{i} \in\left\{s_{1}, s_{2}, \ldots, s_{n}\right\} \cup\left\{u_{1}, u_{2}, \ldots, u_{n}\right\} .
\end{array}
$$

If $h_{i} \notin\left\{s_{1}, s_{2}, \ldots, s_{n}\right\} \cup\left\{u_{1}, u_{2}, \ldots, u_{n}\right\}$, it is obvious that $v\left([\widetilde{S}]_{h}\right)=v\left([\widetilde{T}]_{h}\right)=0$.

Thus, $v\left([\widetilde{S}]_{h}\right)=v\left([\widetilde{T}]_{h}\right)$ for any $h \in[0,1]$.

Addition, if $v\left([\widetilde{S}]_{h}\right)=v\left([\widetilde{T}]_{h}\right)$ for any $h \in[0,1]$, then, by the definition of $v^{\mathrm{Cav}}$, it is easy to see that

$$
v(\widetilde{S})=v(\widetilde{T}) .
$$

\section{The Fuzzy Core of Games with Concave Integral Form}

Now we extend imputation to fuzzy imputation so that it will be available for games with fuzzy coalitions.

Let us suppose that the fuzzy coalitions $\widetilde{U}$ and $\widetilde{S}$ have fuzzy coalition variables $u=\left(u_{1}, u_{2}, \ldots, u_{n}\right)$ and $s=\left(s_{1}, s_{2}, \ldots, s_{n}\right)$, respectively. Then we define fuzzy coalition $\widetilde{S} \widetilde{U} \in L(N)$ which is restricted on $\widetilde{U}$ by

$$
\widetilde{S}_{i}^{U}(j)= \begin{cases}u_{i}, & \text { if } j=i, \\ s_{j}, & \text { otherwise. }\end{cases}
$$

It is obvious that $\widetilde{S}$ is a fuzzy coalition with the fuzzy coalition variable $s^{u}=\left(s_{1}, s_{2}, \ldots, s_{i-1}, u_{i}, s_{i+1}, s_{n}\right)$. When $\widetilde{S} \in$ $P(N)$, then the fuzzy coalition variable $s=(1,1, \ldots, 1)$; then

$$
\widetilde{S}_{i}^{\widetilde{U}}(j)= \begin{cases}u_{i}, & \text { if } j=i, \\ 1, & \text { otherwise. }\end{cases}
$$

Proposition 21. For $i \in N$ and $\widetilde{U} \in L(N)$ with fuzzy coalition variable $u=\left(u_{1}, u_{2}, \ldots, u_{n}\right), \widetilde{S} \subseteq \widetilde{T} \subseteq N \backslash\{i\}$; then

(1) $(\widetilde{S} \cup i)^{\widetilde{U}} \cap \widetilde{T}^{\widetilde{U}}=\widetilde{S}^{\widetilde{U}}$.

(2) $(\widetilde{S} \cup i)^{\widetilde{U}} \cup \widetilde{T}^{\widetilde{U}}=\widetilde{T}^{\widetilde{U}} \cup i^{\widetilde{U}}=\widetilde{T}^{\widetilde{U}}$.

Proof. Denote the fuzzy coalition variables of $\widetilde{S}$ and $\widetilde{T}$ by $t=$ $\left(t_{1}, t_{2}, \ldots, t_{n}\right)$ and $s=\left(s_{1}, s_{2}, \ldots, s_{n}\right)$, respectively. For any $i \in$ $N$, if $\widetilde{S} \subseteq \widetilde{T}$, then $s_{i} \leq t_{i}$ :

$$
(\widetilde{S} \cup i)_{i}^{\widetilde{U}}(j)= \begin{cases}u_{i}, & j=i, \\ s_{j}, & \text { otherwise. }\end{cases}
$$


So the fuzzy coalition variable of $(\widetilde{S} \cup i)_{i}^{\widetilde{U}}$ is $\left(s_{1}, s_{2}, \ldots, s_{i-1}\right.$, $\left.u_{i}, s_{i+1}, s_{n}\right)$, and

$$
\widetilde{T}_{i}^{\widetilde{U}}(j)= \begin{cases}u_{i}, & \text { if } j=i \\ t_{j}, & \text { otherwise. }\end{cases}
$$

We have

$$
\begin{aligned}
(\widetilde{S} \cup i)^{\widetilde{U}} \cap \widetilde{T}^{\widetilde{U}} & = \begin{cases}u_{i}, & j=i, \\
s_{j} \wedge t_{j}, & \text { otherwise }\end{cases} \\
& = \begin{cases}u_{i}, & j=i, \\
s_{j}, & \text { otherwise. }\end{cases}
\end{aligned}
$$

Therefore, the fuzzy coalition variable of $(\widetilde{S} \cup i)^{\widetilde{U}} \cap \widetilde{T}^{\widetilde{U}}$ is $\left(s_{1}, s_{2}, \ldots, s_{i-1}, u_{i}, s_{i+1}, s_{n}\right)$. In other words,

$$
(\widetilde{S} \cup i)^{\widetilde{U}} \cap \widetilde{T}^{\widetilde{U}}=\widetilde{S}^{\widetilde{U}}
$$

For $\widetilde{S} \subseteq \widetilde{T}$, we also note that $(\widetilde{S} \cup i)^{\widetilde{U}}=\widetilde{S}^{\widetilde{U}} \cup i^{\widetilde{U}}$; then

$$
(\widetilde{S} \cup i)^{\widetilde{U}} \cup \widetilde{T}^{\widetilde{U}}=\widetilde{T}^{\widetilde{U}} \cup i^{\widetilde{U}}=\widetilde{T}^{\widetilde{U}}
$$

Proposition 22. A game $v \in G_{C a v}(N, v)$ is fuzzy convex; then, for any $i \in N, \widetilde{U} \in L(N)$, and $\widetilde{S} \subseteq \widetilde{T} \subseteq N \backslash\{i\}$, the game $v^{\text {Cav }}$ satisfies

$$
v^{\operatorname{Cav}}\left(\widetilde{S}^{\widetilde{U}} \cup i^{\widetilde{U}}\right)-v^{\operatorname{Cav}}\left(\widetilde{S}^{\widetilde{U}}\right) \leq v^{\operatorname{Cav}}\left(\widetilde{T}^{\widetilde{U}} \cup i^{\widetilde{U}}\right)-v^{\operatorname{Cav}}\left(\widetilde{T}^{\widetilde{U}}\right) .
$$

Proof. From Proposition 21, we have $(\widetilde{S} \cup i)^{\widetilde{U}} \cap \widetilde{T}^{\widetilde{U}}=\widetilde{S}^{\widetilde{U}}$ and $(\widetilde{S} \cup i)^{\widetilde{U}} \cup \widetilde{T}^{\widetilde{U}}=\widetilde{T}^{\widetilde{U}} \cup i^{\widetilde{U}}=\widetilde{T}^{\widetilde{U}}$. Due to the convexity of $v^{\mathrm{Cav}}$,

$$
v^{\mathrm{Cav}}\left(\widetilde{T}^{\widetilde{U}} \cup i^{\widetilde{U}}\right)+v^{\mathrm{Cav}}\left(\widetilde{S}^{\widetilde{U}}\right) \geq v^{\mathrm{Cav}}\left(\widetilde{S}^{\widetilde{U}} \cup i^{\widetilde{U}}\right)+v^{\mathrm{Cav}}\left(\widetilde{T}^{\widetilde{U}}\right) .
$$

Definition 23. A function $y: L(u) \rightarrow R_{+}^{n}$ is said to be imputation for a fuzzy game $v \in G_{\text {Cav }}(N, v)$ in fuzzy coalition $\widetilde{U} \in L(N)$ with fuzzy coalition variable $u=\left(u_{1}, u_{2}, \ldots, u_{n}\right)$, if

(1) $y_{i}(\widetilde{U})=0, \forall i \notin \operatorname{Supp}(\widetilde{U})$,

(2) $\sum_{i \in N} y_{i}(\widetilde{U})=v^{\mathrm{Cav}}(\widetilde{U})$,

(3) $y_{i}(\widetilde{U}) \geq v^{\operatorname{Cav}}\left(i^{\widetilde{U}}\right)$,

where $y_{i}(\widetilde{U})=\left(y_{1}(\widetilde{U}), y_{2}(\widetilde{U}), \ldots, y_{n}(\widetilde{U})\right)$.

We take the notation $\widetilde{E}\left(v^{\mathrm{Cav}}\right)(\widetilde{U})$ for the set of all imputations of the fuzzy game $v \in G_{\mathrm{Cav}}(N, v)$ on the restricted fuzzy coalition $\widetilde{U} \in L(N)$.

Note that the definition above is also suitable to crisp games. Butnariu [13] and Tsurumi et al. [3] have also proposed the imputation concepts, but these definitions are different from the definition above.

Generally, characteristic functions of games with fuzzy coalitions are difficult to describe clearly in practice so that many fuzzy game models constructed their characteristic function by aggregating one of the crisp games. It means that the game with fuzzy coalition can be represented by a mapping from the characteristic functions of the crisp games to that of the game with fuzzy coalitions, such as Owen fuzzy game, Butnariu fuzzy game, Tsurumi et al. fuzzy game, and the new fuzzy game proposed in this paper.

In the following, we will give another solution for games with fuzzy coalitions, that is, the fuzzy core. At first, we extend the core of crisp game as the imputations for game with fuzzy coalitions.

Definition 24. Let $\widetilde{U} \in L(N)$. The fuzzy core for a game $v \in$ $G_{\text {Cav }}(N, v)$ in the restricted fuzzy coalition $\widetilde{U}$ is the convex set $\widetilde{C}\left(v^{\mathrm{Cav}}\right)(\widetilde{U})$, that is,

$$
\begin{aligned}
& \widetilde{C}\left(v^{\mathrm{Cav}}\right)(\widetilde{U}) \\
& =\left\{y \in R_{+}^{n} \mid \sum_{i \in N} y_{i}=v^{\mathrm{Cav}}(\widetilde{U}),\right. \\
& \left.\sum_{i \in \operatorname{Supp}\left(\widetilde{S}^{\widetilde{U}}\right)} y_{i} \geq v^{\operatorname{Cav}}\left(\widetilde{S}^{\widetilde{U}}\right), \forall \widetilde{S} \in L(\widetilde{U})\right\} .
\end{aligned}
$$

After defining the fuzzy core for a game $v \in G_{\mathrm{Cav}}(N, v)$, we define an excess of a fuzzy coalition similar to that of a crisp coalition.

Letting $\widetilde{U} \in L(N), \widetilde{S} \in L(\widetilde{U})$, and the imputation vector $y=\left\{y_{1}, y_{2}, \ldots, y_{n}\right\}$ for $\widetilde{U}$, an excess for the fuzzy coalition $\widetilde{S} \widetilde{U}$ with respect to the imputation $y$ is denoted by

$$
\widetilde{e}_{\mathrm{Cav}}(\widetilde{S}, y)=v^{\mathrm{Cav}}\left(\widetilde{S}^{\widetilde{U}}\right)-\sum_{i \in \operatorname{Supp}\left(\widetilde{S}^{\widetilde{U}}\right)} y_{i} .
$$

In this way, the fuzzy core of a game $v \in G_{\mathrm{Cav}}(N, v)$ on restricted fuzzy coalition $\widetilde{U}$ can also be considered as the set of all imputation $y$ and all the excess functions are not positive; that is,

$$
\begin{aligned}
\widetilde{C} & \left(v^{\mathrm{Cav}}\right)(\widetilde{U}) \\
& =\left\{y \in \widetilde{E}\left(v^{\text {Cav }}\right)(\widetilde{U}) \mid \widetilde{e}_{\mathrm{Cav}}(\widetilde{S}, y) \leq 0, \forall \widetilde{S} \in L(\widetilde{U})\right\} .
\end{aligned}
$$

Actually, crisp cooperative games, as a special case of cooperative games with fuzzy coalitions, have the excess for $S \in P(N)$; that is,

$$
e(S, y)=v(S)-\sum_{i \in S} y_{i}
$$

where $y=\left\{y_{1}, y_{2}, \ldots, y_{n}\right\}$ is an imputation of $v \in G_{0}(N, v)$. 

by

So the core of a game $v \in G_{0}(N, v)$ can also be represented

$$
\begin{gathered}
C(v)(N)=\{y \in E(v)(N) \mid e(S, y) \leq 0, \\
\forall S \in P(N)\} .
\end{gathered}
$$

It is easy to see that the fuzzy core of game $v \in G_{\mathrm{Cav}}(N, v)$ expressed by (46) is a general form of core for crisp game $v \in$ $G_{0}(N, v)$ identified by $(48)$.

Theorem 25. Let fuzzy coalition $\widetilde{U} \in L(N)$; if $v \in G_{\text {Cav }}(N, v)$ is convex, then $\widetilde{C}\left(v^{\text {Cav }}\right)(\widetilde{U})$ is nonempty.

Proof. Let $v \in G_{\text {Cav }}(N, v)$, let $\widetilde{U} \in L(N)$, and let $\sigma$ be a permutation of the player set $N$; that is, let $Q=$ $\{\sigma(1), \sigma(2), \ldots, \sigma(n)\}$ be a permutation of $N$.

Define a subset $B_{i}^{\sigma}$ of $Q$ by

$$
B_{i}^{\sigma}=\{j \in N \mid \sigma(j)<\sigma(i)\}
$$

Note that $B_{i}^{\sigma}$ is actually the set which precedes $i$ with respect to the order $\sigma$.

We can also define a vector $x^{\sigma}=\left\{x_{1}^{\sigma}, x_{2}^{\sigma}, \ldots, x_{n}^{\sigma}\right\}$, where the factor $x_{i}^{\sigma}(\forall i \in N)$ is $x_{i}^{\sigma}=v^{\mathrm{Cav}}\left(\left(B_{i}^{\sigma} \cup i\right)^{\widetilde{U}}\right)-v^{\mathrm{Cav}}\left(\left(B_{i}^{\sigma}\right)^{\widetilde{U}}\right)$.

In fact, the vector $x^{\sigma}=\left\{x_{1}^{\sigma}, x_{2}^{\sigma}, \ldots, x_{n}^{\sigma}\right\} \in \widetilde{C}\left(v^{\mathrm{Cav}}\right)(\widetilde{U})$.

For one thing,

$$
\sum_{i \in N} x_{i}^{\sigma}=\sum_{i \in N}\left[v^{\mathrm{Cav}}\left(\left(B_{i}^{\sigma} \cup i\right)^{\widetilde{U}}\right)-v^{\mathrm{Cav}}\left(\left(B_{i}^{\sigma}\right)^{\widetilde{U}}\right)\right]=v^{\mathrm{Cav}}(N)^{\widetilde{U}} .
$$

For another thing, we take any subset

$$
\widetilde{S}=\left\{i_{k} \in N|k=1,2, \ldots,| \widetilde{S} \mid\right\} \in L(\widetilde{U})
$$

such that $\sigma\left(i_{1}\right)<\sigma\left(i_{2}\right)<\cdots<\sigma\left(i_{|\widetilde{S}|}\right)$. We have

$$
\left\{i_{1}, i_{2}, \ldots, i_{j-1}\right\} \subseteq \widetilde{S} \subseteq B_{i_{j}}^{\sigma} \quad(j=1,2, \ldots,|\widetilde{S}|)
$$

By Proposition 22,

$$
\begin{aligned}
& v^{\mathrm{Cav}}\left(\left(B_{i_{j}}^{\sigma} \cup i_{j}\right)^{\widetilde{U}}\right)-v^{\mathrm{Cav}}\left(\left(B_{i_{j}}^{\sigma}\right)^{\widetilde{U}}\right) \\
& \geq v^{\operatorname{Cav}}\left(\left\{i_{1}, i_{2}, \ldots, i_{j-1}, i_{j}\right\}^{\widetilde{U}}\right)-v^{\operatorname{Cav}}\left(\left\{i_{1}, i_{2}, \ldots, i_{j-1}\right\}^{\widetilde{U}}\right) .
\end{aligned}
$$

Therefore, $v \in G_{\mathrm{Cav}}(N, v)$ is convex.

For any $j$, by summing the above inequalities, we have that

$$
\begin{aligned}
& v^{\mathrm{Cav}}\left(\left(B_{i_{j}}^{\sigma} \cup i_{j}\right)^{\widetilde{U}}\right)-v^{\mathrm{Cav}}\left(\left(B_{i_{j}}^{\sigma}\right)^{\widetilde{U}}\right) \\
& \geq v^{\mathrm{Cav}}\left(\left\{i_{1}, i_{2}, \ldots, i_{j-1}, i_{j}\right\}^{\widetilde{U}}\right)-v^{\mathrm{Cav}}\left(\left\{i_{1}, i_{2}, \ldots, i_{j-1}\right\}^{\widetilde{U}}\right) .
\end{aligned}
$$

Then,

$$
\begin{aligned}
& v^{\operatorname{Cav}}\left(\left(B_{i}^{\sigma} \cup i\right)^{\widetilde{U}}\right)-v^{\operatorname{Cav}}\left(\left(B_{i}^{\sigma}\right)^{\widetilde{U}}\right) \\
& \geq v^{\operatorname{Cav}}\left(\left\{i_{1}, i_{2}, \ldots, i_{|\widetilde{S}|-1}, i_{|\widetilde{S}|}\right\}^{\widetilde{U}}\right) \\
& \quad-v^{\operatorname{Cav}}\left(\left\{i_{1}, i_{2}, \ldots, i_{|\widetilde{S}|-1}\right\}^{\widetilde{U}}\right), \\
& \sum_{i \in \operatorname{Supp}\left(\widetilde{S}^{\widetilde{U}}\right)} x_{i}^{\sigma}\left(\sum_{i \in \operatorname{Supp}\left(\widetilde{S}^{\widetilde{U}}\right)}\left[v^{\operatorname{Cav}}\left(\left(B_{i}^{\sigma} \cup i\right)^{\widetilde{U}}\right)-v^{\operatorname{Cav}}\left(\left(B_{i}^{\sigma}\right)^{\widetilde{U}}\right)\right]\right. \\
& \geq \sum_{i \in \operatorname{Supp}\left(\widetilde{S}^{\widetilde{U}}\right)}\left[v^{\operatorname{Cav}}\left(\left\{i_{1}, i_{2}, \ldots, i_{|\widetilde{S}|-1}, i_{|\widetilde{S}|}\right\}^{\widetilde{U}}\right)\right. \\
& =v^{\operatorname{Cav}}\left(\widetilde{S}^{\widetilde{U}}\right) . \\
& \left.-v^{\operatorname{Cav}}\left(\left\{i_{1}, i_{2}, \ldots, i_{|\widetilde{S}|-1}\right\}^{\widetilde{U}}\right)\right]
\end{aligned}
$$

Hence, $x^{\sigma} \in \widetilde{C}\left(v^{\mathrm{Cav}}\right)(\widetilde{U})$; that is to say, $\widetilde{C}\left(v^{\mathrm{Cav}}\right)(\widetilde{U})$ is nonempty.

Now, let us pay more attention to the calculating method of the fuzzy core for the game given by the concave integral.

Let $v \in G_{\mathrm{Cav}}(N, v)$ and a fuzzy coalition $\widetilde{U} \in L(N)$; the excess $\widetilde{e}_{\text {Cav }}(\widetilde{S}, y)$ of the fuzzy restricted coalition $\widetilde{S}^{\widetilde{U}}$ with respect to the imputation $y$ can be calculated by

$$
\begin{aligned}
\widetilde{e}_{\mathrm{Cav}}(\widetilde{S}, y) & =v^{\mathrm{Cav}}\left(\widetilde{S}^{\widetilde{U}}\right)-\sum_{i \in \operatorname{Supp}\left(\widetilde{S^{U}}\right)} y_{i} \\
& =\int^{\mathrm{Cav}} s^{u} d v-\sum_{i \in \operatorname{Supp}\left(\widetilde{S}^{\widetilde{U}}\right)} y_{i},
\end{aligned}
$$

where

$$
\int^{\mathrm{Cav}} s^{u} d v=\max \sum_{A_{i} \subseteq L(\widetilde{U})} \alpha_{A_{i}} v\left(A_{i}\right)
$$

$$
\text { s.t. }\left\{\begin{array}{l}
\sum_{i=1}^{|\widetilde{U}|} \alpha_{A_{i}} 1_{A_{i}}=s^{u}, \\
A_{i} \subseteq L(\widetilde{U}) \quad(i=1,2, \ldots,|\widetilde{U}|), \\
\alpha_{A_{i}} \in\left\{s_{1}, s_{2}, \ldots, s_{i-1}, u_{i}, s_{i+1}, s_{n}\right\} .
\end{array}\right.
$$


The above inequality is

$$
\begin{gathered}
\int_{A_{i} \subseteq L(\widetilde{U})}^{\text {Cav }} s^{u} d v=\max \sum_{A_{i}} v\left(A_{i}\right) \\
=\max \sum_{A_{i} \subseteq \operatorname{Supp}\left(\widetilde{S^{U}}\right)} \alpha_{A_{i}} v\left(A_{i}\right) \\
\text { s.t. }\left\{\begin{array}{l}
|\widetilde{U}| \\
\sum_{i=1} \alpha_{A_{i}} 1_{A_{i}}=s^{u}, \\
A_{i} \subseteq \operatorname{Supp}(\widetilde{S}) \quad(i=1,2, \ldots,|\widetilde{U}|), \\
\alpha_{A_{i}} \in\left\{s_{1}, s_{2}, \ldots, s_{i-1}, u_{i}, s_{i+1}, s_{n}\right\} .
\end{array}\right.
\end{gathered}
$$

Then, the fuzzy core of $v \in G_{\text {Cav }}(N, v)$ can be represented by

$$
\begin{aligned}
& \widetilde{C}\left(v^{\mathrm{Cav}}\right)(\widetilde{U}) \\
& =\left\{y \in R_{+}^{n} \mid \sum_{i \in N} y_{i}=\int^{\mathrm{Cav}} s d v,\right. \\
& \left.\quad \sum_{i \in \operatorname{Supp}\left(\widetilde{S^{U}}\right)} y_{i} \geq \int^{\mathrm{Cav}} s^{u} d v, \text { for any } \widetilde{S} \in L(\widetilde{U})\right\} .
\end{aligned}
$$

That is to say that the fuzzy core of game $v \in G_{\mathrm{Cav}}(N, v)$ can also be represented by

$$
\begin{gathered}
\widetilde{C}\left(v^{\text {Cav }}\right)(\widetilde{U}) \\
=\left\{y \in R_{+}^{n} \mid \sum_{i \in N} y_{i}=\max \sum_{i=1}^{|\widetilde{U}|} \alpha_{A_{i}} v\left(A_{i}\right),\right. \\
\sum_{i \in \operatorname{Supp}(\widetilde{S})} y_{i} \geq \max \sum_{i=1}^{|\widetilde{U}|} \alpha_{A_{i}(\widetilde{U})} v\left(A_{i}(\widetilde{U})\right), \\
\text { for any } \widetilde{S} \in L(\widetilde{U})\},
\end{gathered}
$$

where $A_{i}(\widetilde{U}) \subseteq \operatorname{Supp}(\widetilde{S} \widetilde{U})$ and $\alpha_{A_{i}(\widetilde{U})} \in\left\{s_{1}, s_{2}, \ldots, s_{i-1}, u_{i}\right.$, $\left.s_{i+1}, s_{n}\right\}$.

It is not easy to find that $\widetilde{C}\left(v^{\mathrm{Cav}}\right)(\widetilde{U}) \geq \widetilde{C}\left(v^{\mathrm{Cav}}\right)\left(\widetilde{S}^{\widetilde{U}}\right)$.

Proposition 26. Let $v \in G_{C a v}(N, v)$ and a fuzzy coalition $\widetilde{U} \in L(N)$; then, for all $\widetilde{U}$-restricted fuzzy coalition $\widetilde{S} \widetilde{U} \in L(\widetilde{U})$ denoted by $O\left(\widetilde{S}^{\widetilde{U}}\right), v^{C a v}\left(O\left(\widetilde{S}^{\widetilde{U}}\right)\right)=v^{\text {Cav }}(\widetilde{U})$.
Proof. One has the following:

$$
\begin{aligned}
& \int^{\text {Cav }} s^{u} d v=\max \sum_{A_{i} \subseteq L(\widetilde{U})} \alpha_{A_{i}} v\left(A_{i}\right)=v^{\operatorname{Cav}}\left(\widetilde{S}^{\widetilde{U}}\right) \\
& \text { s.t. }\left\{\begin{array}{l}
\sum_{i=1}^{\left|\widetilde{S}^{\widetilde{U}}\right|} \alpha_{A_{i}} 1_{A_{i}}=s^{u}, \\
A_{i} \subseteq L(\widetilde{U}) \quad\left(i=1,2, \ldots,\left|\widetilde{S}^{\widetilde{U}}\right|\right), \\
\alpha_{A_{i}} \in\left\{s_{1}, s_{2}, \ldots, s_{i-1}, u_{i}, s_{i+1}, s_{n}\right\} .
\end{array}\right.
\end{aligned}
$$

Hence,

$$
\begin{gathered}
\int_{A^{u} \text { Cav }}^{u} d v=\max \sum_{A_{i} \subseteq L(\widetilde{U})} \alpha_{A_{i}} v\left(A_{i}\right) \\
\text { s.t. }\left\{\begin{array}{l}
\sum_{A_{i} \subseteq \operatorname{Supp}\left(\widetilde{S}^{\widetilde{U}}\right)} \alpha_{A_{i}} v\left(A_{i}\right) \\
\sum_{i=1}^{|\widetilde{U}|} \alpha_{A_{i}} 1_{A_{i}}=s^{u}, \\
A_{i} \subseteq \operatorname{Supp}(\widetilde{U}) \quad(i=1,2, \ldots,|\widetilde{U}|), \\
\alpha_{A_{i}} \in\left\{s_{1}, s_{2}, \ldots, s_{i-1}, u_{i}, s_{i+1}, s_{n}\right\} .
\end{array}\right.
\end{gathered}
$$

For all $i \in N$, we get

$$
v^{\mathrm{Cav}}\left(O\left(\widetilde{S}^{\widetilde{U}}\right)\right)=\int^{\mathrm{Cav}} O\left(s^{u}\right) d v=\max \sum_{A_{i} \subseteq L(\widetilde{U})} \alpha_{A_{i}} v\left(A_{i}\right)
$$

$$
\text { s.t. }\left\{\begin{array}{l}
\sum_{i=1}^{|\widetilde{U}|} \alpha_{A_{i}} 1_{A_{i}}=u, \\
A_{i} \subseteq L(\widetilde{U}) \quad(i=1,2, \ldots,|\widetilde{U}|), \\
\alpha_{A_{i}} \in\left\{u_{1}, u_{2}, \ldots, u_{n}\right\} .
\end{array}\right.
$$

Thus,

$$
v^{\mathrm{Cav}}\left(O\left(\widetilde{S}^{\widetilde{U}}\right)\right)=\int^{\mathrm{Cav}} u d v=v^{\mathrm{Cav}}(\widetilde{U})
$$

Theorem 27. Let $v \in G_{C a v}(N, v)$ and a fuzzy coalition $\widetilde{U} \in$ $L(N)$; if all the games defined on fuzzy coalition $\widetilde{S}$ are convex, then $\widetilde{C}\left(v^{\operatorname{Cav}}\right)(\widetilde{U}) \neq \phi$, and

$$
\begin{aligned}
& \widetilde{C}\left(v^{C a v}\right)(\widetilde{U}) \\
& =\left\{y \in R_{+}^{n} \mid \sum_{k \in N} y_{k}=\int^{C a v} x d v,\right. \\
& \left.\forall x \in \widetilde{C}\left(v^{C a v}\right)\left(\widetilde{S}^{\widetilde{U}}\right), \text { for any } \widetilde{S} \in L(\widetilde{U})\right\} .
\end{aligned}
$$


Proof. It is obvious that $\widetilde{C}\left(v^{\mathrm{Cav}}\right)(\widetilde{S} \widetilde{U}) \neq \phi$. denote

Let $\widetilde{U} \in L(N)$ and any $x=\left(x_{1}, x_{2}, \ldots, x_{n}\right) \in \widetilde{C}\left(v^{\mathrm{Cav}}\right)\left(\widetilde{S}^{\widetilde{U}}\right)$;

$$
\begin{aligned}
y_{k}=\max \sum_{A_{i} \subseteq L(\widetilde{U})} \alpha_{A_{i}} x_{k}\left(A_{i}\right), \quad(k=1,2, \ldots, n), \\
\sum_{k \in N} y_{k}=\sum_{k \in N} \max \sum_{A_{i} \subseteq L(\widetilde{U})} \alpha_{A_{i}} x_{k}\left(A_{i}\right) \\
=\max \sum_{k \in N_{A_{i} \subseteq L(\widetilde{U})}} \alpha_{A_{i}} x_{k}\left(A_{i}\right) \\
=\max \sum_{A_{i} \subseteq L(\widetilde{U})} \alpha_{A_{i}} \sum_{k \in N} x_{k}\left(A_{i}\right) \\
=\sum_{A_{i} \subseteq L(\widetilde{U})} \alpha_{A_{i}} v\left(A_{i}\right)=v^{\mathrm{Cav}}(\widetilde{U}) .
\end{aligned}
$$

Also,

$$
\begin{aligned}
\sum_{k \in \operatorname{Supp}\left(\widetilde{S^{U}}\right)} y_{k} & =\sum_{k \in \operatorname{Supp}\left(\widetilde{S}^{\widetilde{U}}\right)} \max \sum_{i=1}^{\left|\widetilde{S}^{\widetilde{U}}\right|} \alpha_{A_{i}} x_{k}\left(A_{i}\right) \\
& =\max \sum_{i=1}^{\left|\widetilde{S}^{\widetilde{U}}\right|} \sum_{k \in \operatorname{Supp}\left(\widetilde{S}^{\widetilde{U}}\right)} \alpha_{A_{i}} x_{k}\left(A_{i}\right) \\
& =\max \sum_{i=1}^{\left|\widetilde{S}^{\widetilde{U}}\right|} \alpha_{A_{i}} \sum_{i \in \operatorname{Supp}\left(\widetilde{S}^{\widetilde{U}}\right)} x_{k}\left(A_{i}\right) \\
& \geq \max \sum_{i=1}^{\left|\widetilde{S}^{\widetilde{U}}\right|} \alpha_{A_{i}} v^{\operatorname{Cav}}\left(\widetilde{S}^{\widetilde{U}}\right) \geq v^{\operatorname{Cav}}(\widetilde{S}) .
\end{aligned}
$$

Hence, $\widetilde{C}\left(v^{\mathrm{Cav}}\right)(\widetilde{U}) \neq \phi$.

Next, we will illustrate that $\forall z \in \widetilde{C}\left(v^{\mathrm{Cav}}\right)(\widetilde{U})$ can be calculated by

$$
z=\int^{\mathrm{Cav}} x d v
$$

Let $\underline{x}_{j}=\min \left\{x_{j} \mid \forall x \in \widetilde{C}\left(v^{\mathrm{Cav}}\right)\left(\widetilde{S}^{\widetilde{U}}\right), j \in N\right\}$ and $\bar{x}_{j}=$ $\max \left\{x_{j} \mid \forall x \in \widetilde{C}\left(v^{\mathrm{Cav}}\right)\left(\widetilde{S}^{\widetilde{U}}\right), j \in N\right\}$.

Suppose that $\underline{x}=\left\{\underline{x}_{1}, \underline{x}_{2}, \ldots, \underline{x}_{n}\right\}$ and $\bar{x}=\left\{\bar{x}_{1}, \bar{x}_{2}, \ldots, \bar{x}_{n}\right\}$. Also let

$$
\begin{aligned}
& \bar{S}=\left\{j \in N \mid z_{j}>\max \sum_{A_{i} \subseteq L(\widetilde{U})} \alpha_{A_{i}} \bar{x}_{j}\left(A_{i}\right)\right\}, \\
& \underline{S}=\left\{j \in N \mid z_{j}<\max \sum_{A_{i} \subseteq L(\widetilde{U})} \alpha_{A_{i}} \underline{x}_{j}\left(A_{i}\right)\right\} .
\end{aligned}
$$

Now we will prove that the two sets $\bar{S}$ and $\underline{S}$ are empty.

If there exists $j \in N$ such that $z_{j}$ cannot be expressed by (69), then there are only two cases for $z_{j}$ that should be considered.
Case a. $z_{j}<\max \sum_{A_{i} \subseteq L(\widetilde{U})} \alpha_{A_{i}} \underline{x}_{j}\left(A_{i}\right)$.

Case b. $z_{j}>\max \sum_{A_{i} \subseteq L(\widetilde{U})} \alpha_{A_{i}} \bar{x}_{j}\left(A_{i}\right)$.

In Case a, since $z \in \widetilde{C}\left(v^{\mathrm{Cav}}\right)(\widetilde{U})$, we have

$$
v^{\mathrm{Cav}}\left(\underline{S}^{\widetilde{U}}\right) \leq \sum_{j \in \operatorname{Supp}\left(\underline{S}^{\widetilde{U}}\right)} z_{j}<\max \sum_{A_{i} \subseteq L(\widetilde{U})} \alpha_{A_{i}} \underline{x}_{j}\left(A_{i}\right) .
$$

Hence,

$$
\begin{aligned}
& 0<\sum_{j \in \operatorname{Supp}\left(\underline{S}^{\widetilde{U}}\right)} \max \sum_{A_{i} \subseteq L(\widetilde{U})} \alpha_{A_{i}} \underline{x}_{j}\left(A_{i}\right)-v^{\mathrm{Cav}}\left(\underline{S}^{\widetilde{U}}\right) \\
& =\max \sum_{A_{i} \subseteq L(\widetilde{U})} \alpha_{A_{i}} \sum_{j \in \operatorname{Supp}\left(\underline{S}^{\widetilde{U}}\right)} \underline{x}_{j}\left(A_{i}\right)-v^{\mathrm{Cav}}\left(\underline{S}^{\widetilde{U}}\right) \\
& =\max \sum_{A_{i} \subseteq L(\widetilde{U})} \alpha_{A_{i}} \sum_{j \in \operatorname{Supp}\left(\underline{S}^{\widetilde{U}}\right)} \underline{x}_{j}\left(A_{i}\right) \\
& -\max \sum_{A_{i} \subseteq L(\widetilde{U})} \alpha_{A_{i}} \sum_{j \in \operatorname{Supp}\left(\underline{S}^{\widetilde{U}}\right)} v\left(A_{i}\right) \\
& =\max \sum_{A_{i} \subseteq L(\widetilde{U})} \alpha_{A_{i}}\left(\sum_{j \in \operatorname{Supp}\left(\underline{S}^{\widetilde{U}}\right)} \underline{x}_{j}\left(A_{i}\right)-\sum_{j \in \operatorname{Supp}\left(\underline{S}^{\widetilde{U}}\right)} v\left(A_{i}\right)\right) \\
& =\max \sum_{A_{i} \subseteq L(\widetilde{U})} \alpha_{A_{i}}\left(\sum_{j \in \operatorname{Supp}\left(\underline{S}^{\widetilde{U}}\right)} v(\{j\})-\sum_{j \in \operatorname{Supp}\left(\underline{S}^{\widetilde{U}}\right)} v\left(A_{i}\right)\right) \\
& <0 \text {. }
\end{aligned}
$$

This is contrary to $v \in G_{\mathrm{Cav}}(N, v)$.

In Case b,

$$
\begin{aligned}
\sum_{j \in N} z_{j}> & \sum_{j \in S} \max \sum_{A_{i} \subseteq L(\widetilde{U})} \alpha_{A_{i}} \bar{x}_{j}\left(A_{i}\right) \\
& +\sum_{j \in N \backslash S} \max \sum_{A_{i} \subseteq L(\widetilde{U})} \alpha_{A_{i}} x_{j}\left(A_{i}\right) \\
\geq & \sum_{j \in N} \max \sum_{A_{i} \subseteq L(\widetilde{U})} \alpha_{A_{i}} x_{j}\left(A_{i}\right)=v^{\mathrm{Cav}}(\widetilde{U}) .
\end{aligned}
$$

We note that this is also contrary to the fact $z \in \widetilde{C}\left(v^{\mathrm{Cav}}\right)(\widetilde{U})$.

Since neither Case a nor Case $\mathrm{b}$ is true, therefore, any $z \in$ $\widetilde{C}\left(v^{\text {Cav }}\right)(\widetilde{U})$ can be given by $(69)$.

Remark 28. Convexity is a sufficient condition but not necessary for the nonempty fuzzy core of the game $v \in G_{\text {Cav }}(N, v)$.

Proposition 29. Let $v \in G_{\text {Cav }}(N, v)$, a fuzzy coalition $\widetilde{U} \in$ $L(N)$, and $x \in \widetilde{C}\left(v^{C a v}\right)(\widetilde{U})$. If fuzzy coalition $\widetilde{T} \subseteq \widetilde{U}$, then $x$ satisfies

$$
\sum_{i \in N} x_{i} \geq v^{\operatorname{Cav}}(\widetilde{T})
$$


Proof. Let $x \in \widetilde{C}\left(v^{\mathrm{Cav}}\right)(\widetilde{U})$. If $\widetilde{T} \subseteq \widetilde{U}$, by (29), we get that

$$
v^{\mathrm{Cav}}(\widetilde{T}) \leq v^{\mathrm{Cav}}(\widetilde{U}) .
$$

Because $x \in \widetilde{C}\left(v^{\mathrm{Cav}}\right)(\widetilde{U})$, we have

$$
\sum_{i \in N} x_{i}=v^{\mathrm{Cav}}(\widetilde{U}) .
$$

Hence,

$$
\sum_{i \in N} x_{i} \geq v^{\mathrm{Cav}}(\widetilde{T})
$$

Example 30. We continue to consider Example 16.

Let set $N=\{1,2,3\}, v(1)=v(2)=v(3)=2, v(1,3)=8$, $v(1,2)=9, v(2,3)=5$, and $v(1,2,3)=10$. Suppose the fuzzy coalition $s=(1,0.4,0.6)$; then

$$
\begin{aligned}
v^{\mathrm{Cav}}(\widetilde{S}) & =s_{2} \times v(1,2)+s_{3} \times v(1,3) \\
& =0.4 \times 9+0.6 \times 8=8.4 .
\end{aligned}
$$

Let $x^{\{1,2\}} \in C(\{1,2\})$ and $x^{\{1,3\}} \in C(\{1,3\})$; then

$$
\begin{aligned}
& \left\{x^{\{1,2\}} \mid x_{1}^{\{1,2\}}+x_{2}^{\{1,2\}}\right. \\
& \left.\quad=v(1,2), x_{1}^{\{1,2\}} \geq v(1), x_{2}^{\{1,2\}} \geq v(2)\right\}, \\
& \left\{x^{\{1,3\}} \mid x_{1}^{\{1,3\}}+x_{3}^{\{1,3\}}\right. \\
& \left.\quad=v(1,3), x_{1}^{\{1,3\}} \geq v(1), x_{3}^{\{1,3\}} \geq v(3)\right\} .
\end{aligned}
$$

Let $x=\left(x_{1}, x_{2}, x_{3}\right) \in \widetilde{C}\left(v^{\text {Cav }}\right)(\widetilde{S})$; then we have

$$
\begin{gathered}
x_{1}=0.4 x_{1}^{\{1,2\}}+0.6 x_{1}^{\{1,3\}}, \\
x_{2}=0.4 x_{2}^{\{1,2\}}, \quad x_{3}=0.6 x_{3}^{\{1,3\}} .
\end{gathered}
$$

Hence,

$$
\begin{aligned}
& C^{\mathrm{Cav}}(v)=\left\{x=\left(x_{1}, x_{2}, x_{3} \mid x_{1}+x_{2}+x_{3}=8.4\right)\right\} \\
& \text { s.t. }\left\{\begin{array}{l}
x_{1}=0.4 x_{1}^{\{1,2\}}+0.6 x_{1}^{\{1,3\}} \\
x_{2}=0.4 x_{2}^{\{1,2\}} \\
x_{3}=0.6 x_{3}^{\{1,3\}} \\
x_{1}^{\{1,3\}}+x_{3}^{\{1,3\}}=8 \\
x_{1}^{\{1,2\}}+x_{2}^{\{1,2\}}=9, \\
x_{1}^{\{1,3\}}, x_{3}^{\{1,3\}}, x_{1}^{\{1,2\}}, x_{2}^{\{1,2\}} \geq 2 .
\end{array}\right.
\end{aligned}
$$

Obviously, $(4,2,2.4) \in \widetilde{C}\left(v^{\mathrm{Cav}}\right)(\widetilde{S})$.

\section{Conclusions}

At first, a new integral representation for games with fuzzy coalitions is introduced. We know that the concave integral with fuzzy capacities enables one to calculate the collective profits of a team when the available information is limited to a few events.

The value of games with Choquet integral is only calculated on nest coalitions so that it is smaller than that of our models. It is natural that the concave integral representation for fuzzy game can be regarded as a general form of cooperative game with fuzzy coalitions.

The fuzzy core is an important content in fuzzy cooperative games, so we have defined the fuzzy core of the games with fuzzy coalitions and have given the nonempty condition of the fuzzy core. The fuzzy core is based on the assumption that the total value of a fuzzy coalition will be allocated to the players whose participation rate is larger than zero. The method of fuzzy core will help us have a better understanding of the fuzzy core and avoid the complicated computation process. As in the classical game, the relationship between core and the Shapley value always coincides. In future research, we will further investigate the relationship between fuzzy core and the fuzzy Shapley function.

\section{Conflict of Interests}

The authors declare that they do not have any commercial or associative interests that represent a conflict of interests in connection with the submitted work. There are no professional or other personal interests that can inappropriately influence the paper.

\section{Acknowledgments}

This research was supported by the National Natural Science Foundation, China (Grant no. 71271029), and Education of Humanities and Social Science Research on Self-Fund Project, China (Grant no. 11YJE630003). The authors would like to express their deep gratitude to the referee for his/her valuable comments and suggestions.

\section{References}

[1] J. P. Aubin, "Coeur et valeur des jeux flous à paiements latéraux," Comptes Rendus Hebdomadaires des Séances de l'Académie des Sciences A, vol. 279, pp. 891-894, 1974.

[2] D. Butnariu, "Fuzzy games: a description of the concept," Fuzzy Sets and Systems, vol. 1, no. 3, pp. 181-192, 1978.

[3] M. Tsurumi, T. Tanino, and M. Inuiguchi, "A shapley function on a class of cooperative fuzzy games," European Journal of Operational Research, vol. 129, no. 3, pp. 596-618, 2001.

[4] D. Granot, "Cooperative games in stochastic characteristic function form," Management Science, vol. 23, no. 6, pp. 621-630, 1977.

[5] F. R. Fernandez, J. Puerto, and M. J. Zafra, "Cores of stochastic cooperative games," International Game Theory Review, vol. 4, pp. $265-280,2002$. 
[6] L. A. Zadeh, "Fuzzy sets," Information and Control, vol. 8, no. 3, pp. 338-353, 1965.

[7] M. Mareš, "Weak arithmetics of fuzzy numbers," Fuzzy Sets and Systems, vol. 91, no. 2, pp. 143-153, 1997.

[8] D. Dubois and H. Prade, "Fundamentals of fuzzy sets," in The Handbooks of Fuzzy Stes Series, Kluver Academic Publishers, Boston, Mass, USA, 2000.

[9] M. Mareš, "Fuzzy coalition structures," Fuzzy Sets and Systems, vol. 114, no. 1, pp. 23-33, 2000.

[10] M. Mareš, Fuzzy Cooperative Games: Cooperation with Vague Expectations, Physica, New York, NY, USA, 2001.

[11] M. Mareš and M. Vlach, "Linear coalitional games and their fuzzy extensions," International Journal of Uncertainty, Fuzziness and Knowlege-Based Systems, vol. 9, no. 3, pp. 341-354, 2001.

[12] S. Borkotokey, "Cooperative games with fuzzy coalitions and fuzzy characteristic functions," Fuzzy Sets and Systems, vol. 159, no. 2, pp. 138-151, 2008.

[13] D. Butnariu, "Stability and Shapley value for an n-persons fuzzy game," Fuzzy Sets and Systems, vol. 4, no. 1, pp. 63-72, 1980.

[14] D. Butnariu and T. Kroupa, "Shapley mappings and the cumulative value for n-person games with fuzzy coalitions," European Journal of Operational Research, vol. 186, no. 1, pp. 288-299, 2008.

[15] S. Tijs, R. Brânzei, S. Ishihara, and S. Muto, "On cores and stable sets for fuzzy games," Fuzzy Sets and Systems, vol. 146, no. 2, pp. 285-296, 2004.

[16] J. Dow and S. Werlang, "Uncertainty aversion, risk aversion, and the optimal choice of portfolio," Econometrica, vol. 60, pp. 197204, 1992.

[17] J. Dow and S. R. D. C. Werlang, "Nash equilibrium under knightian uncertainty: breaking down backward induction," Journal of Economic Theory, vol. 64, no. 2, pp. 305-324, 1994.

[18] R. J. Weber, "Games in coalitional form," in Handbook of Game Theory, R. J. Aumann and S. Hart, Eds., vol. 2, Elsevier, Amsterdam, The Netherlands, 1994.

[19] Y. Azrieli and E. Lehrer, "Extendable cooperative games," Journal of Public Economic Theory, vol. 9, no. 6, pp. 1069-1078, 2007.

[20] E. Lehrer, "A new integral for capacities," Economic Theory, vol. 39, no. 1, pp. 157-176, 2009. 


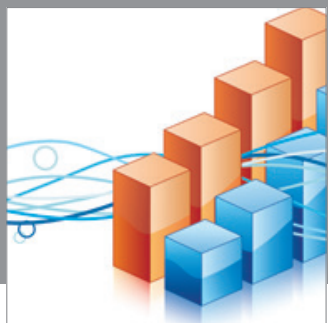

Advances in

Operations Research

mansans

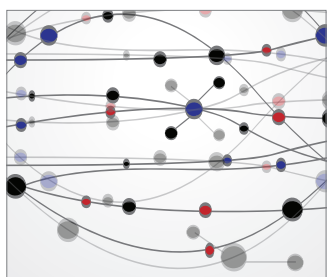

The Scientific World Journal
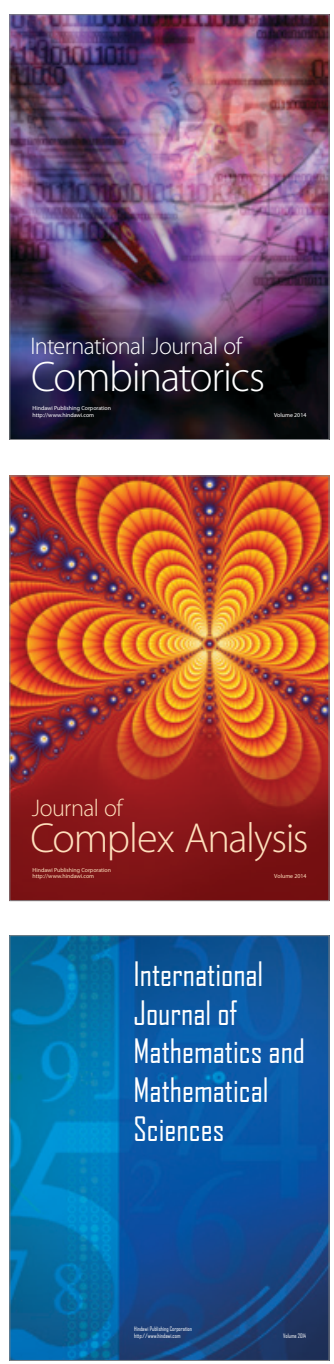
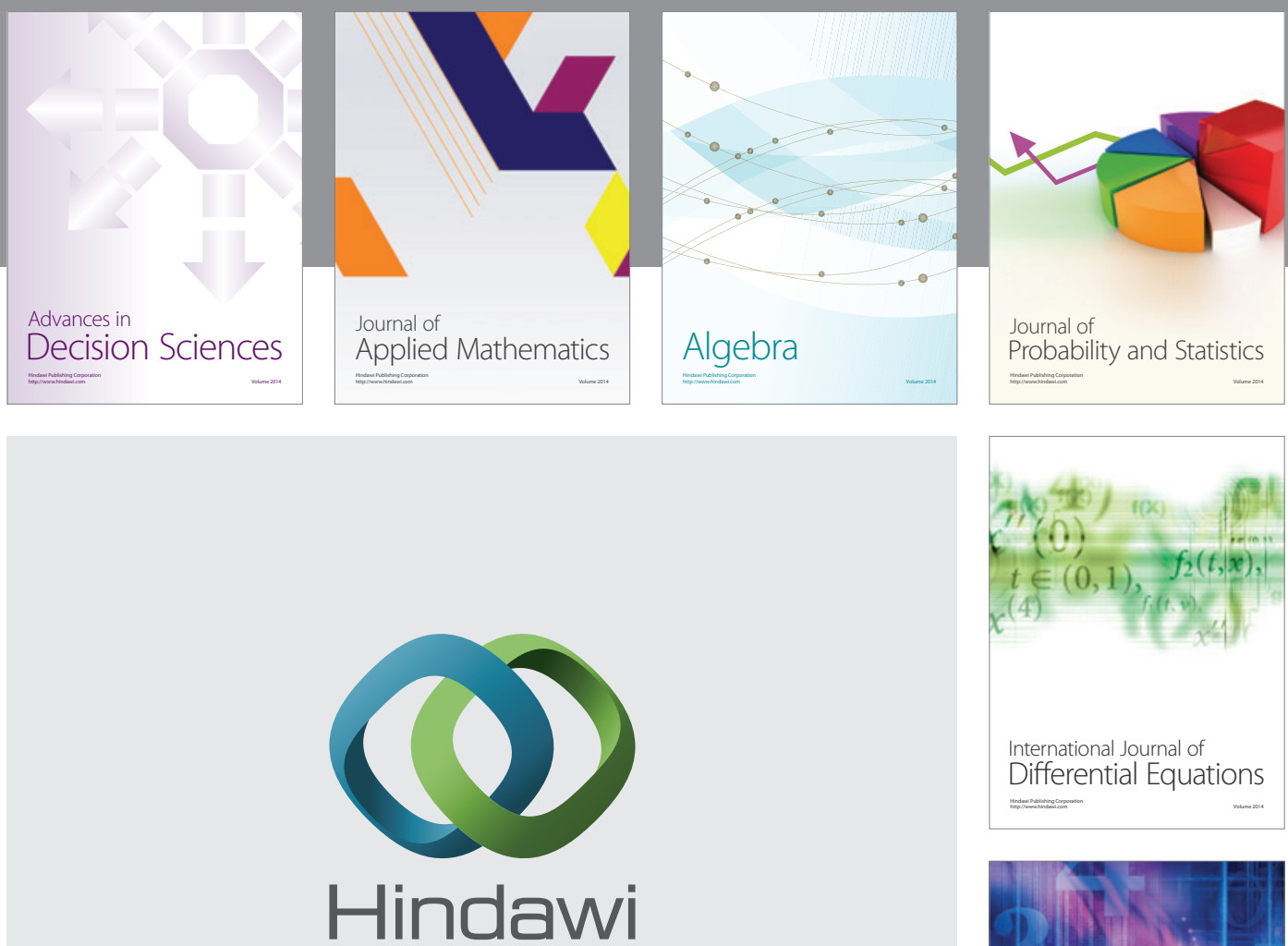

Submit your manuscripts at http://www.hindawi.com
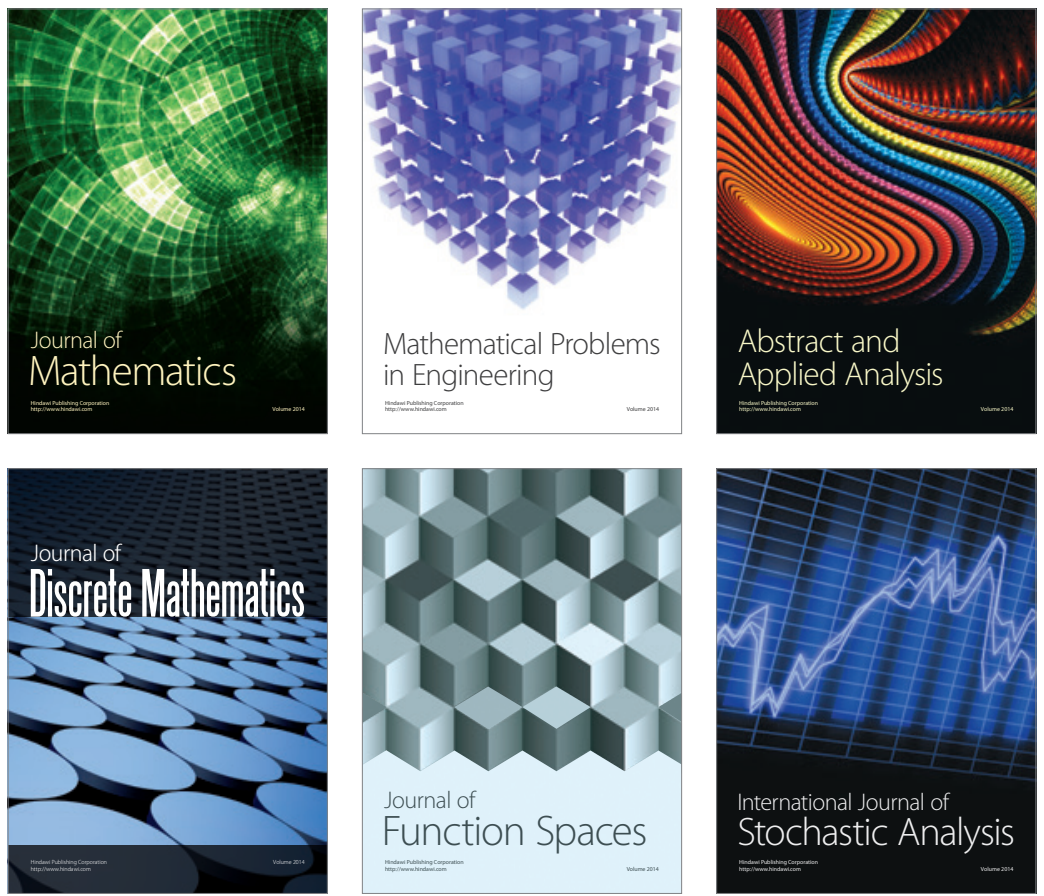

Journal of

Function Spaces

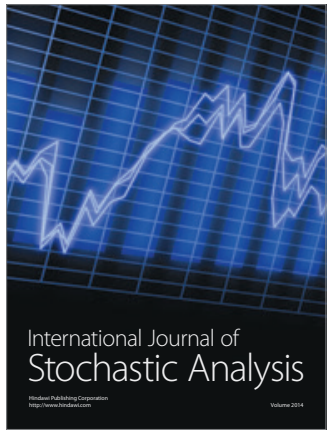

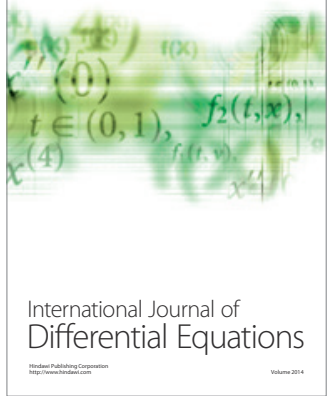
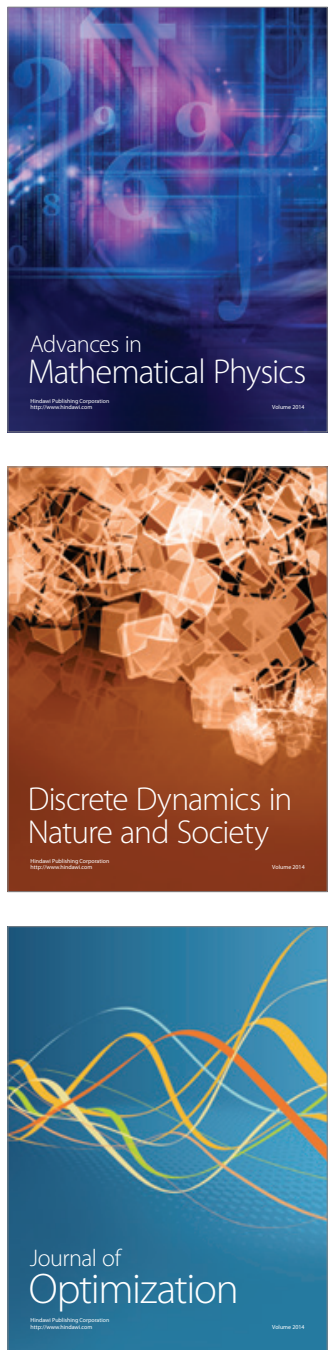